\title{
The product of the pilQ gene is essential for the biogenesis of type IV pili in Neisseria gonorrhoeae
}

\author{
Sandra L. Drake and Michael Koomey* \\ Department of Microbiology and Immunology, \\ University of Michigan Medical School, Ann Arbor, \\ Michigan 48109-0620, USA.
}

\section{Summary}

The product of the Nelsseria gonorrhoeae omc gene possesses reglons homologous to those found in members of a protein superfamily that are assoclated with the translocation of proteins and DNA-protein complexes across the outer membrane. Amongst its protein homologues, Omc has higher overall homology to PilQ, which is required for type IV pilus expression in Pseudomonas aeruginosa, and OrfE, which is required for sequence-specific DNA uptake by Haemophilus influenzae. The function of Omc, however, is unknown and gonococcal omc mutants have not been described. We constructed gonococcal mutants expressing truncated forms of the protein, and found that these mutants are severely defective for both pilus expression and competence for natural transformation. To be consistent with pre-existing pilus gene nomenclature, we have redesignated the gene pilQ instead of omc, and its product, PilQ instead of Omc. The MS11 gene was sequenced and found to differ from the DNA sequence reported for that of another gonococcal strain; these differences were associated with a repeated DNA element, suggesting a genetic basis for structural variation in PIIQ. The results also show that PilQ ${ }^{-}$mutants are distinct from previously described gonococcal pilus-assembly mutants and $P$. aeruginosa PilQ $^{-}$mutants by virtue of their expression of rare pilus filaments. Taking these data into account, PilQ is proposed to function in the terminal steps of organelle biogenesis by acting as a pilus channel or pore.

\section{Introduction}

The expression of pili by Neisseria gonorrhoeae, the causative agent of gonnorhoea, appears to be of paramount importance to the ability of the organism to infect its human host. Evidence for the critical role of gonococcal

Received 17 April, 1995; revised 16 August, 1995; accepted 21 August, 1995. "For correspondence. Tel. (313) 763 3672; Fax (313) 7643562.

(C) 1995 Blackwell Science Ltd pili can be found in the invariable recoveries of piliated organisms from primary cultures, the failure of nonpiliated mutants to cause urethral infection in male volunteers (Swanson and Koomey, 1989) and the capacity of these structures to undergo antigenic variation (Swanson et al., 1987; Haas and Meyer, 1986; Virji et al., 1983). High-frequency antigenic variation of the pilin subunit appears to preclude the efficacy of pilus-based vaccines and may be responsible for the inability of gonococcal infection to engender a protective immune response. Based on the conserved structural features of its pilin subunit protein, gonococcal pili are included in a rapidly expanding family of related fimbrial appendages termed type IV pili. Pili of this type are expressed by a number of other significant Gram-negative human pathogens as well as commensal species capable of causing opportunistic infection. The former group includes Neisseria meningitidis (Hermodson et al., 1978), Vibrio cholerae (Shaw and Taylor, 1990) and enteropathogenic Escherichia coli (Giron et al., 1991), and, like the case in gonococci, these related pili appear to act as colonization factors. Gonococcal pili appear to promote infectivity by mediating attachment to mucosal epithelium (Swanson, 1973; Heckels, 1989; Rudel et al., 1992). Pilus expression is also correlated with other phenotypes which may be relevant to host-parasite biology including competence for transtormation (Seifert et al., 1990; Zhang et al., 1992), bacterial autoagglutination (Swanson et al., 1971), and twitching motility (Swanson, 1978), which is a non-flagellar based form of movement across solid surfaces. The basis for the associations between these phenotypes and piliation is not well understood.

We have focused on studies directed toward understanding the mechanisms of gonococcal pilus biogenesis with the rationale that such efforts might lead to a clearer understanding of pilus structure and function, and the identification of new targets for chemotherapeutic or immunoprophylactic intervention. In past studies, domains and residues of the pilin subunit itself that are required for pilus assembly have been delineated (Zhang et al., 1992; Koomey et al., 1991). More recently, a single gonococcal gene cluster was shown to carry three genes, pilD, pilF and pilG (Lauer et al., 1993), whose products are essential for pilus assembly (Freitag et al., 1995; Tønjum et al., 1995). These genes encode a pre-pilin peptidase, a soluble protein with consensus nucleotide binding motifs and a polytopic membrane protein of unknown activity 
respectively. Protein homologues of each of these have previously been shown to be components of the Type II secretion system which encompasses the processes of two-step export of toxins and hydrolases by Gramnegative organisms (Pugsley, 1993), type IV pilus assembly (Nunn et al., 1990) and DNA uptake in Bacillus subtilus (Albano et al., 1989).

The products of the gonococcal pilus assembly genes we have characterized to date are all predicted to be localized to the cytoplasm or the cytoplasmic membrane. A $110 \mathrm{kDa}$ protein, PilC, that copurifies with the gonococcal pilin subunit and its corresponding gene have been identified (Jonsson et al., 1991) and mutants failing to express PilC were reported to have a significant reduction in piliation. Although it was claimed that PilC fractionated as an outer membrane protein, it is difficult to envision what role it serves since neither the gene sequence nor its derived primary structure has been published or submitted to standard databases. By characterizing nonpiliated transposon insertion mutants of $P$. aeruginosa, Martin et al. (1993) identified the pilus assembly gene pirQ and found that the predicted amino acid sequence of PIIQ showed homology to the pIV/PuID/YscC protein superfamily (Huang et al., 1992). Members of this family possess $\mathrm{N}$-terminal signal sequences, share sequence relatedness in a $\sim 200$-amino-acid span at their C-termini, and function in protein-export processes across the outer membrane (Salmond and Reeves, 1993). Surprisingly, these translocation events involve widely disparate substrates including filamentous phage, type IV pilin/pili, toxins and hydrolases (Type II secretion; Pugsley, 1993) and proteins lacking typical signal sequences (Type III secretion; Michiels and Cornelis, 1991).

Most members of the $\mathrm{pIV} / \mathrm{PuID} / \mathrm{YscC}$ protein superfamily have been identified by genetic studies, and relatively little is known about their cellular localization and function. Notable exceptions to this situation can be found in studies of $\mathrm{f1}$ pIV and PuID (required for pullulanase secretion), which have shown that a significant proportion of these molecules fractionate with the outer membrane (d'Enfert et al., 1989; Brissette and Russel, 1990). MxiD (a homologue required for secretion of the Shigella invasion-plasmid antigens) is found in the detergent-insoluble membrane fraction suggesting that it too is localized, in part, to the outer membrane (Allaoui et al., 1993). In addition to their carboxy-terminal shared sequence identities and predicted amphipathic $\beta$-sheet conformations, many of the homologues induce the $p s p$ operon when expressed in E. coli (Russel and Kasmierczak, 1993; Possot et al., 1992). As this response can be triggered by other membrane-perturbing stimuli (Brissette et al., 1990; Carison and Silhavy, 1993), it was proposed that the homologues might activate $p s p$ expression in a similar manner (Russel and Kazmierczak, 1993). Clues as to the functions of the homologues are most clearly found in the filamentous phage assembly/secretion system where it has been demonstrated that the conserved carboxy-terminal domain of pIV mediates its insertion into the membrane (Russel and Kasmierczak, 1993) and that, in that milieu, plV is capable of forming a homomultimer composed of 10-12 subunits (Kazmierczak et al., 1994). Furthermore, non-functional pIV missense mutants altered at a highly conserved residue in the carboxy-terminal domain (P375) fail to multimerize despite their membrane localization (Russel, 1994). On the basis of these data, it was proposed that multimerized piV (and by analogy, similar structures composed of its homologues) might form a gated channel or pore through which substrates are translocated.

Martin et al. (1993) noted that the derived primary structure of Pseudomonas aeruginosa PilQ protein was most related to that of the gonococcal Omc (Outer membrane protein-macromolecular complex) protein, a polypeptide not previously recognized as belonging to the pIV/PulD/ YscC superfamily. These two proteins are $30 \%$ identical and blocks of similarity are distributed across their entire lengths. Omc was originally characterized as an abundant gonococcal outer membrane protein with a propensity to form homo-multimers of 10-12 monomers that could be dissociated only after reduction and alkylation (Newhall et al., 1980). Interest in Omc as a gonococcal vaccine component was fostered by the observations that it appeared to account for $10 \%$ of the outer membrane protein mass (Newhall et al., 1980), that it was conserved in strains of diverse origin, and that it was suceptible to iodination (Hansen and Wilde, 1984) and proteolysis (Tsai et al., 1989) in whole cells. The omc gene was subsequently cloned and sequenced but the function of Omc has yet to be determined (Tsai $e t$ al., 1989). Given these cumulative findings, we asked whether Omc might be a gonococcal pilus-assembly factor. In this study, we have constructed gonococcal mutants expressing truncated forms of this protein and show that the mutants are grossly deficient in pilus expression and fail to display pilus-associated phenotypes. In light of these results, and to be consistent wtih pre-existing pilus gene nomenclature, we have redesignated the omc gene, pilQ, and its product, PilQ.

\section{Results}

Cloning, expression and sequencing of the MS11 pilQ gene in $\mathrm{E}$. coli

Fragments of the pilQ gene (previously designated omc) have been cloned from Gc strain 2686, but attempts at cloning the complete gene intact were unsuccessful (Tsai et al., 1989). Primers based on the 2686 pilQ gene sequence were used to generate a $1 \mathrm{~kb}$ fragment carrying the $3^{\prime}$ end of the gene by polymerase chain reaction (PCR) 


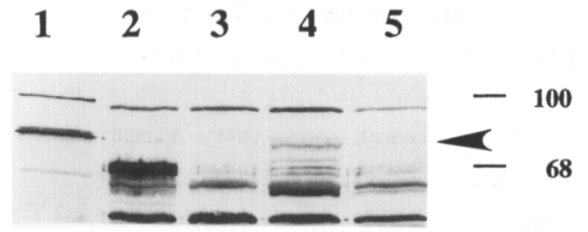

Fig. 1. Detection of PilQ expression in whole-cell lysates of $E$. coli clones. Lanes: 1, N. gonorrhoeae N400; 2, HB101/pCF1; 3, HB101/ $\mathrm{pBE7} ; 4, \mathrm{HB} 101 / \mathrm{pAEB}$; and 5, HB101/pAD8. Note that pBE7 contains only the $3^{\prime}$ end of pilQ and serves as a negative control. The relative migration of PilQ monomers $(75 \mathrm{kDa})$ is denoted by the arrowhead. Gonococcal PilQ polyclonal antiserum was used at 1:1000 dilution. The relative mobilities of molecular mass standards are shown in $\mathrm{kDa}$.

of genomic DNA from strain N400, which is the MS11 variant used in our studies. After sequencing to confirm that it represented the $3^{\prime}$ end of the gene, the PCR product was used as a DNA probe to identify pilQ-containing $E$. coli clones in an MS11-derived genomic cosmid library. Three of the four probe-positive clones grew poorly and when whole-cell lysates were examined by immunoblotting with PilQ antibodies, the poor growth phenotype correlated with expression of an immunoreactive species co-migrating with gonococcal PilQ (Fig. 1). Restriction mapping of the cosmid DNAs showed that the probe-positive, antigennegative clone carried only the $3^{\prime}$ end of the pilQ gene, while the others contained the complete pilQ gene and upstream sequences. The levels of immunoreactive antigen varied in the three positive clones and differences in other reactive species were noted. Given the polyclonal nature of the antiserum, it is not clear whether these other differences correspond to degraded forms of PilQ, other gonococcal proteins encoded by the cosmids, or E. coli proteins induced in response to pilQ expression. The poor growth phenotype also correlated with changes in the cell morphology as seen by the formation of chains of elongated cells (data not shown).

DNA fragments containing overlapping but incomplete parts of the pilQ gene were subcloned from cosmid PAE8 onto high-copy-number plasmids and the entire nucleotide sequence of the MS11 gene was determined. The pilQ open reading frame (ORF) encodes a polypeptide with a molecular weight of 77590 and an isoelectric point of 9.75 . The $\mathrm{N}$-terminal domain has characteristics typical of signal sequences, and the first residue of the mature polypeptide has been shown to be the glycine at residue 25 (Hansen and Wilde, 1984). Comparison of the pilQ nucleotide sequences from strains MS11 and 2686 revealed remarkable differences with significant consequences for PilQ structure (Fig. 2). The MS11 allele contains three additional single nucleotides (inserted after nucleotide positions 500, 503 and 676 of the sequence from strain 2686) and a unique block of 24 nucleotides (inserted after nucleotide position 597 of the sequence from 2686). Consequently, this 204 bp region of the MS11

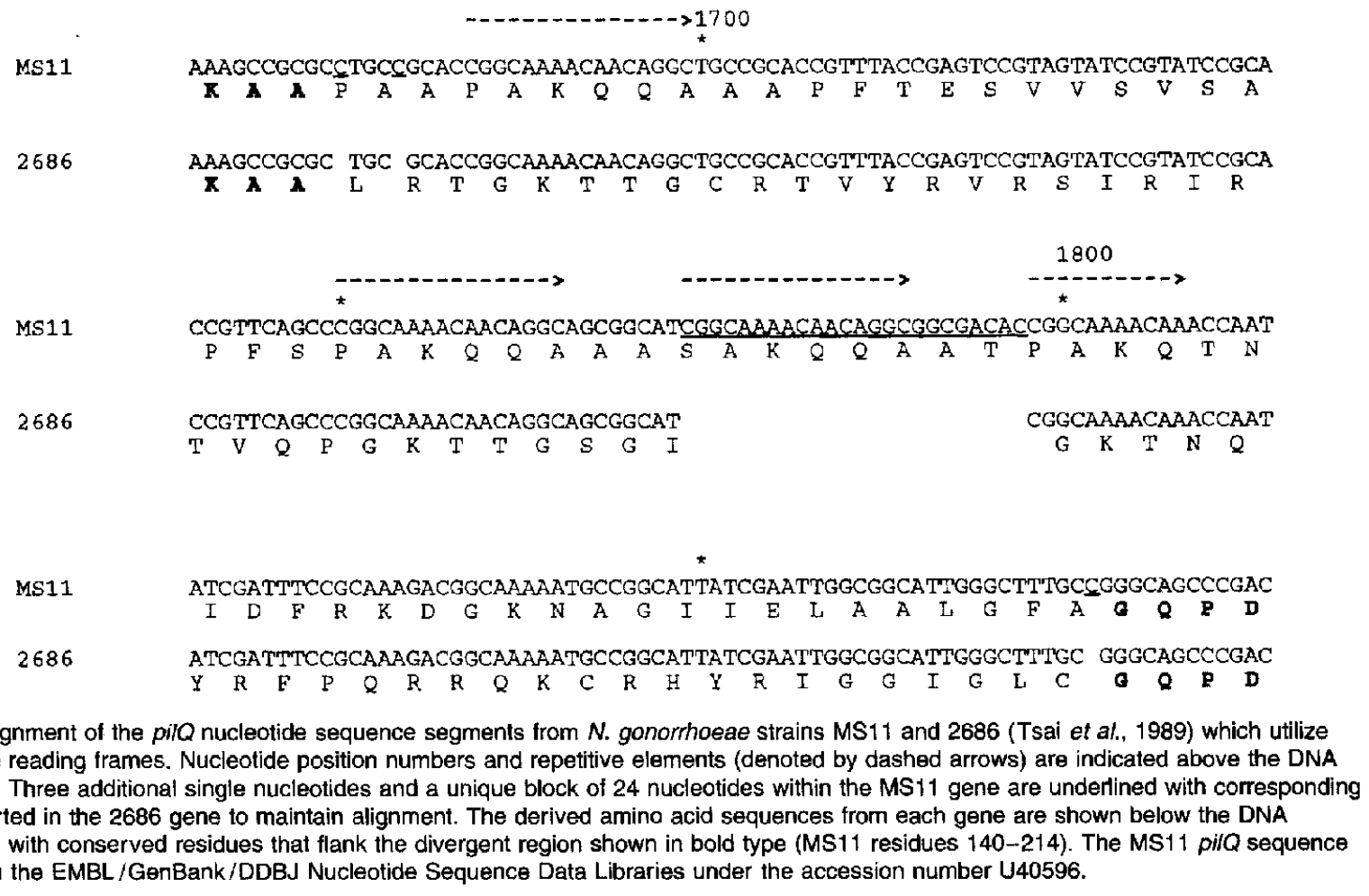
alternative reading frames. Nucleotide position numbers and repetitive elements (denoted by dashed arrows) are indicated above the DNA sequence. Three additional single nucleotides and a unique block of 24 nucleotides within the MS11 gene are underlined with corresponding gaps inserted in the 2686 gene to maintain alignment. The derived amino acid sequences from each gene are shown below the DNA sequence, with conserved residues that flank the divergent region shown in bold type (MS11 residues 140-214). The MS11 pilQ sequence appears in the EMBL/GenBank/DDBJ Nucleotide Sequence Data Libraries under the accession number U40596. 
allele utilizes an alternative reading frame from that of strain 2686. Examination of the unique 24 bp block present in the MS11 allele (position 1774-1797) revealed that it was composed of a $16 \mathrm{bp}$ nucleotide repeat present at positions 1684-1699 and 1750-1765 and an 8bp sequence that bridges it to a fourth subrepeat that contains 11 of the 16 nucleotides (position 1798-1808). These four nucleotide repeat elements are all in the same reading frame and encode the residues PAKQQAA (residues 146-152 and 168-174), AKQQAA (residues 177-187) and PAKQ (residues 184-187). Direct sequencing of PCR products encompassing this region generated from genomic DNA revealed no differences from that derived for the plasmid clones, ruling out the possibility that the MS11 allele differences were a result of rearrangements arising during cloning in $E$. coli.

\section{Construction of PII- mutants}

Defined lesions in pilQ were made in cloned DNAs in $E$. coli, by transposon mutagenesis using a mini-Tnerm element and insertion of an ermC gene cassette. Three transposon-insertion mutants, predicted to disrupt the conserved carboxy terminal domain of PilQ, and one which mapped $3^{\prime}$ of the ORF, were selected for further study (Fig. 6). DNA sequencing showed that the precise sites of insertion were situated in the codons for mature residues L399 (pGQ1), D447 (pGQ4), and K560 (pGQ5),

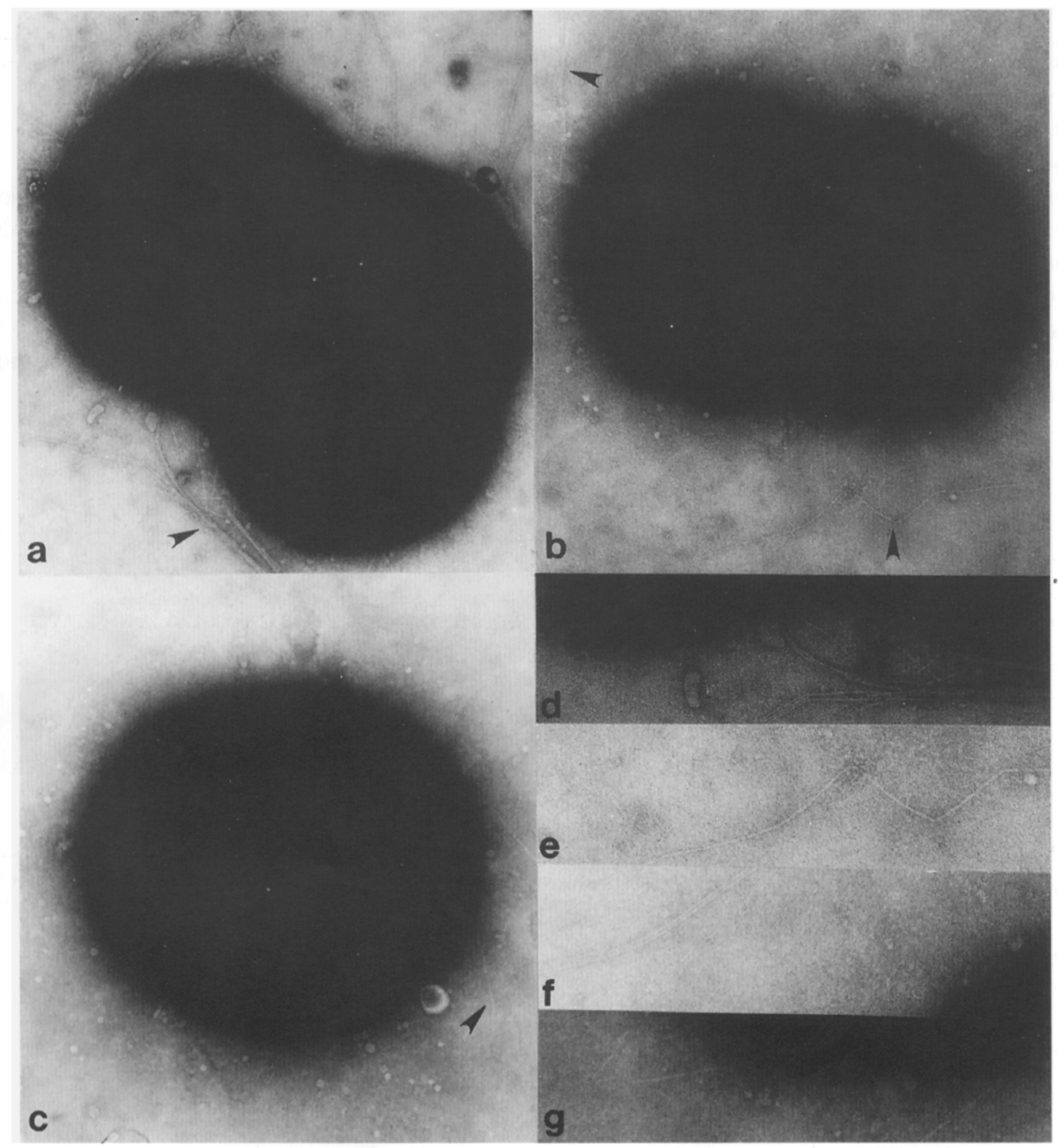

Fig. 3. Transmission electron micrographs of $N$. gonorrhoeae strains: a, N400; b, GQ1; c, GQ3; d, enlargement of N400 pilus filaments from (a); $e$ and $f$, enlargement of rare GQ1 pilus filaments from (b); and g, enlargement of rare GQ3 pilus filaments from (c). Arrowheads in (a-c) denote the location of pilus filaments enlarged in $(d-g)$. Magnification $31500 \times(a-c)$.

(C) 1995 Blackwell Science Ltd, Molecular Microbiology, 18, 975-986 
while the fourth insertion (pGQ6) was 45 bp downstream of the termination codon. pGQ6 was modified so as to carry a frameshift mutation in pilQ within the codon for mature residue D421, by filling in the $5^{\prime}$ overhang of DNA linearized at a single Clal restriction site, followed by intramolecular ligation (creating $p G Q 3$ ). A sixth mutation was constructed by insertion of an ermC gene cassette into this same Clal site (creating PGQ2). These mutations were then moved onto the chromosome of strain $\mathrm{N} 400$ by transformation, as described in the Experimental procedures. After ensuring the correct introduction of the mutations by Southern hybridization and that no changes in pilE (the pilin subunit gene) had been incurred during the process, pilus expression and associated phenotypes were assessed.

\section{Characterization of pilus expression and associated phenotypes in PilQ ${ }^{-}$mutants}

All mutants in which the integrity of the pilQORF was compromised were deficient in autoagglutination, a property closely associated with pilus expression. These phenotypic alterations in patterns of growth were manifested as a flat, spreading colony type on solid media, and the failure to aggregate in liquid cultures. Transformants arising from introduction of the downstream transposon-insertion mutation had growth characteristics indistinguishable from that of N400. In the case of erythromycin-resistant transformants arising from pGQ3, two approximately equally represented populations were found, differing in autoagglutination phenotypes. The non-autoagglutinating transformants were shown to correspond to those in which the linked pilQ frameshift mutation had been crossed into the genome, while those which were phenotypically unaltered had only incorporated the downstream transposon insertion.

When examined by transmission electron microscopy (TEM), cells of strains N400 and GQ6 were associated with abundant levels of pili that were often present as discrete bundles of laterally aggregated filaments, while all pilQ mutants were devoid of pilus filaments (Fig. 3). However, careful examination revealed that rare pilus filaments could be reproducibly detected on grids of all of these mutants. The filaments seen in the mutants were identical in diameter, average length and morphology to those found in the wild-type strain and, in some instances, were also seen to aggregate laterally. In view of this finding, we reexamined pilD, pilF and pilG mutants previously characterized as lacking pili (Tonjum et al., 1995; Freitag et al., 1995), but could find no evidence for rare filaments in these specimens. It therefore appeared that the rare pilus filaments were unique to pilQ defects and were not a general property of gonococcal pilus-assembly mutants.

Previous studies have shown that non-piliated mutants are dramatically reduced in competence for natural transformation and that pilus expression appears to be required

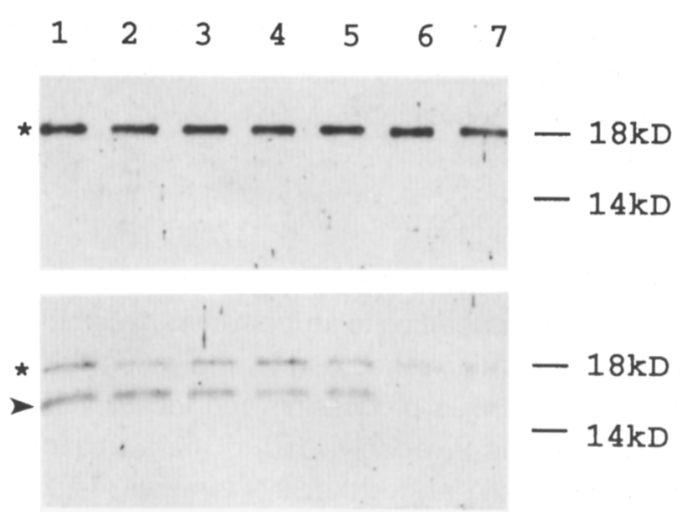

Fig. 4. Detection of pilin antigen in whole-cell lysates (top panel) and concentrated culture supernatants (bottom panel) from wild-type and mutant gonococcal strains by immunoblotting. The filter was reacted with a polyclonal antiserum raised against purified N400 pili. Lanes: 1, GQ1; 2, GQ4; 3, GQ5; 4, GQ2; 5, GQ3; 6, GQ6; and 7, N400. The relative mobilities of mature (pre-pilin peptidase-processed) pilin and S-pilin are denoted by the asterisk and the arrowhead, respectively. The relative mobilities of molecular mass standards are shown in $\mathrm{kDa}$.

for this property (Seifert et al., 1990; Zhang et al., 1992; Tønjum et al., 1995; Freitag et al., 1995). However, antigenic variants with relatively low levels of pili are transformable at frequencies equivalent to those seen for extensively piliated strains (Gibbs et al., 1989). Given that competence for transformation appears to be a sensitive marker for piliation, we examined the relative competence of N400, GQ6 and the isogenic pilQ mutants. All mutants that were dramatically reduced in pilus expression had over a 1000-fold reduction in their transformation competence (Table 1), levels which are equivalent to that found for non-piliated mutants resulting from defects in expression of the pilin subunit as well as from pilD, pilF and pilG gene mutations. Also, as similarly found for these other classes of assembly mutants, the transformants recovered in the $\mathrm{PilQ}^{-}$background retained their defective-pilus phenotype. This finding appeared to rule

Table 1. Phenotypic characteristics of $N$. gonormoeae $\mathrm{N} 400$ and PilQ mutants.

\begin{tabular}{lccc}
\hline Strain & Pili $^{\mathrm{a}}$ & Pilin/S-pilin $^{\mathrm{b}}$ & $\%$ Natural competence $^{c}$ \\
\hline N400 & + & $-1-$ & 100 \\
GQ1 & $-*$ & $+/+$ & $<0.1$ \\
GQ2 & $-^{*}$ & $+/+$ & $<0.1$ \\
GQ3 & $-{ }^{*}$ & $+/+$ & $<0.1$ \\
GQ4 & $-{ }^{*}$ & $+/+$ & $<0.1$ \\
GQ5 & $-{ }^{*}$ & $+/+$ & $<0.1$ \\
GQ6 & + & $-1-$ & 100 \\
\hline
\end{tabular}

a. Determined by transmission electron microscopy. ${ }^{*}$ rare pilus filaments observed.

b. Detection in the supernatant by Western blotting with anti-pilin antibodies.

c. Determined using the DNA transformation assay under recAinduced conditions. 
out the possibility that the pilus filaments associated with PilQ mutants reflected the presence of rare piliated revertants within that population.

\section{Pilin expression, processing and localization in PilQ- mutants}

Diverse mutations leading to an inability to assemble gonococcal pili or to diminished levels of piliation have been linked to altered pilin processing and localization. Pilusassembly defects invariably lead to the formation of Spilin (truncated pilin lacking the $39 \mathrm{~N}$-terminal residues present in mature pilin), some of which is recovered in culture supernatants, while the remainder is cell associated (Freitag et al., 1995; Koomey et al., 1991; Tønjum et al., 1995). With this in mind, we used immunoblotting to assess the

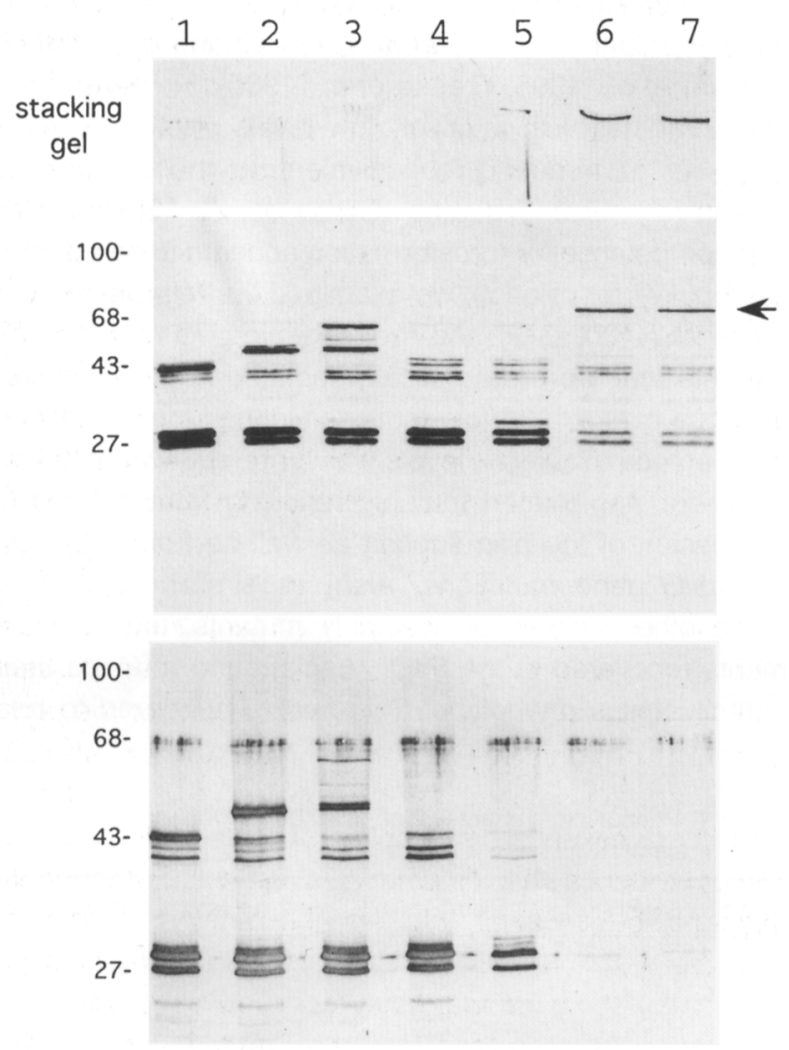

Fig. 5. Detection of PilQ antigen in whole-cell lysates (top and middle panels) and concentrated culture supernatants (bottom panel) from wild-type and mutant gonococcal strains by immunoblotting. The whole cell and concentrated supernatant samples correspond to $50 \mu \mathrm{l}$ and $250 \mu \mathrm{l}$ of the liquid culture, respectively. The filter was reacted with a polyclonal antiserum raised against PilQ purified from strain 2686. Lanes: 1, GQ1; 2, GQ4; 3, GQ5; 4, GQ2; 5, GQ3; 6, GQ6; and 7, N400. The relative mobility of wild-type PilQ (75 kDa) is denoted by the arrowhead. The relative mobilities of molecular mass standards are shown in $\mathrm{kDa}$. status of pilin expression in pilQ mutants. Although the amounts of pilin antigen detected were equivalent in all cases, the patterns of pilin species found were identically altered in the mutants (Fig. 4). The predominant pilin form in mutant whole-cell lysates migrated with a relative mobility characteristic of pre-pilin peptidase (PilD)processed subunits, findings similar to those seen in other assembly mutants (Koomey et al., 1991; Tønjum et al., 1995). Whole-cell lysates of PilQ mutants also contained species of pilin antigen co-migrating with S-pilin found in other assembly mutants but were present at lower levels than in the latter mutants (data not shown). Immunoblotting of concentrated culture supernatants confirmed that all pilQ mutants elaborated S-pilin, but pilin antigen migrating at the position of the predominant species seen in the mutant-cell lysates was also found in the culture supernatants. This finding was again in contrast to results from other assembly mutants where pilin in this form has not been detected. In this way, the levels of mature, intact pilin in culture supernatants appeared to coincide with the presence of rare pilus filaments. In summary, $\mathrm{PilQ}^{-}$mutants are distinguished by (i) the release of mature, intact pilin into culture supernatants, and (ii) their reduced capacity to retain S-pilin in association with cells.

\section{PilQ expression and localization}

Immunoblotting with rabbit antibodies raised against purified PilQ from strain 2686 was employed to examine the expression of PilQ in N400 and the pilQ mutants. The predominant form seen in cell lysates of the wild-type strains migrated with a molecular mass of $75 \mathrm{kDa}$, a finding in agreement with the predicted molecular mass for PilQ. Newhall and colleagues (1980) originally suggested that gonococcal PilQ exists as a homo-multimer with a molecular mass of $800 \mathrm{kDa}$, which only enters standard polyacrylamide gels upon reduction and alkylation. In agreement with this, detectable levels of antibody-reactive material are found at the position of the electrophoresis stacking wells in immunoblats of wild-type samples (Fig. 5, top panel). Minor reactive polypeptides were also seen, the most apparent of which were two with molecular masses of 27 and $30 \mathrm{kDa}$. The $75 \mathrm{kDa}$ form and antigen-containing material in the stacking gel were missing in each of the pilQ mutants and in its place were new fragments whose migration corresponded to the sizes of truncated PilQ species predicted to be encoded by the defective alleles. Increased levels of the 27 and $30 \mathrm{kDa}$ reactive polypeptides were also present in the mutants. Two other lines of evidence support the idea that the 27 and $30 \mathrm{kDa}$ polypeptides, and the other minor species, represent processed (degraded) forms derived from the $\mathrm{N}$-terminal domains of PilQ: (i) identical patterns are seen for all of these strains when rabbit antibodies raised against

(C) 1995 Blackwell Science Ltd, Molecular Microbiology, 18, 975-986 


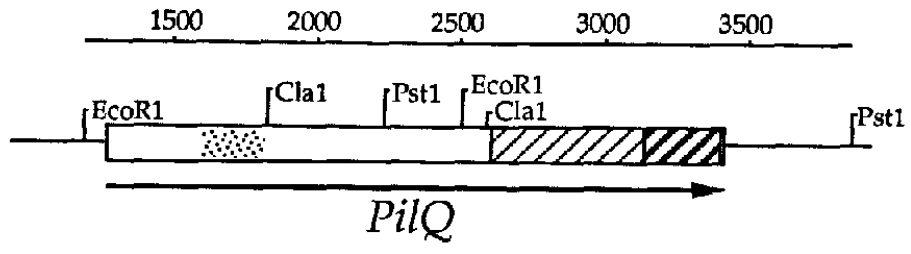

PQ4

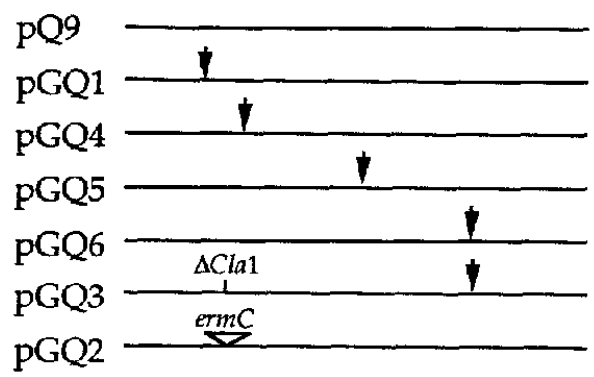

Fig. 6. Physical map of the inserts from relevant plasmids used in this study. The thin and thick striped segments of the pilQ ORF correspond to the regions that are moderately and highly conserved in the plV/YscC/PulD protein superfamily. The stippled segment denotes the region encompassing the altered reading frames found for strains MS11 and 2686 (see Fig. 2). Positions of restrictions sites are noted, as are sites of $\mathrm{m}$-Tnerm transposon insertions. The vector for the pGQ series of plasmids is pHSS6. recombinant meningococcal PilQ expressed in $E$. coli are used (data not shown); and (ii) they are not present in gonococcal mutants with mutations in the $5^{\prime}$ end of pilQ (S. Drake and M. Koomey, in preparation).

The expression of defective forms of PilQ had a profound influence on the cellular localization of the truncated polypeptides themselves. Immunoblotting of the soluble proteins present in concentrated culture supernatants of the mutants revealed patterns that were indistinguishable from that seen in the whole-cell lysates, while culture supernatants of the wild-type strains revealed barely perceptible levels of the 75, 27 and $30 \mathrm{kDa}$ species. Based on the relative intensities seen with equivalent proportions of cells and supernatants from cultures, we estimate that $20 \%$ of the total PilQ antigen in the mutants is shed in an extracellular, soluble form.

The most striking finding from these immunoblot experiments is the dramatic increase in the levels of PilQ antigen in the mutants as indicated by the comparative intensities of the multiple bands in the mutants versus what is seen in the wild-type strains. A simple explanation for this may be that it reflects a redistribution of PilQ antigen which is normally retained in the stacking gel. However, alkylation/ reduction treatment (leading to a loss of antigen from that position in wild-type samples) results in only a moderate increase in levels of the $75 \mathrm{kDa}$ species that does not equal the antigen levels seen in the mutants (data not shown).

\section{Discussion}

The biogenesis of the gonococcal pilus colonization factor and other type IV pili requires the function of a large number of gene products. In this paper, we report the characterization of the pilQ gene and gene product from $N$. gonormoeae strain MS11, and demonstrate that gonococcal mutants expressing truncated PilQ polypeptides are severely compromised in the elaboration of pili and are deficient in pilus-associated phenotypes. The findings provide prima facie evidence that gonococcal PilQ is functionally as well as structurally related to members of the pIV/YscC/PulD superfamily that are essential components of systems in which diverse protein substrates are translocated across bacterial membranes. Cross-linking studies have shown that pIV exists as a homo-multimer, probably composed of 10-12 subunits (Kazmierczak et al., 1994). To our knowledge, gonococcal PilQ is the only other homologue for which the existence of multimers has been demonstrated and, interestingly, the PilQ multimer has also been estimated to be composed of $10-12$ subunits. Studies of pIV mutants specifically altered in Cterminal residues that are highly conserved amongst the homologues indicate that this preserved domain is involved in pIV multimerization and that pIV multimerization is essential for phage assembly/secretion (Russel, 1994). The defects in pilus biogenesis found for the gonococcal mutants comply with those observations whereby the structural integrity of the conserved C-terminal domain of PilQ is essential for function. There is, however, no direct evidence that differentiates between whether the high-molecular-weight PilQ antigen detected within the stacking gel represents a discrete-sized, functional oligomer or merely an aberrant aggregation of the protein.

The increased levels of truncated PilQ antigens observed in the mutants do not appear to reflect a simple redistribution of antigen retarded at the stacking-gel interface in wildtype strains. Although it may, in part, result from an actual increase in pilQ gene expression (transcription) in this background, the curious and poorly understood behaviour of the protein itself raises serious caveats to data interpretation. For example, one cannot discern whether all the PilQ antigen in wild-type strains enters the gel and is 
detectable by immunoblotting or whether a significant portion is lost or unrecoverable for technical reasons. It is worth noting, nonetheless, that increased levels of antigen, its degradation and release in soluble form, are intrinsic properties of $\mathrm{PilQ}^{-}$mutants and not simply consequences of disrupted pilus assembly, because none of these are detected in uncharacterized non-piliated mutants (Hansen and Wilde, 1984) or in pilE, pilD, pilF or pilG mutants (S. Drake and M. Koomey, in preparation).

Kazmierczak and colleagues (1994) have proposed that the pIV multimer, and, by analogy, similar structures formed by the homologues, could form a gated channel or pore that couples phage assembly to export or directs specific protein substrates across the outer membrane. This model is perhaps more readily appreciated in the context of the more classical Type II secretion systems, where the first step entails the localization of toxins and hydrolases to the periplasm (mediated by the sec gene products), while, in the second step, fully formed and matured periplasmic complexes are translocated across the outer membrane. In additon to a pIV-related protein, this latter step requires the function of four components including type IV pseudopilin(s), a pre-pilin peptidase, a soluble protein with consensus nucleotide-binding motifs, and a polytopic inner-membrane protein of unknown function (Whitchurch et al., 1991; Pugsley, 1992; Lory, 1992). Like these other situations where formation of a pilus-like organelle is implied but as yet unsubstantiated, gonococcal pilus expression requires all of the latter components. However, PilQ mutants have unique features that distinguish them from other gonococcal pilus mutants, the most remarkable of which is the presence of rare pilus filaments. This appears to indicate that pilin polymerization (assembly) is still occurring in these mutants and that PilQ is not necessary for this aspect of pilus biogenesis but, rather, may be critical for the extrusion of pilus filaments. However, if each of these suppositions is true, how can we reconcile them with the presence of both soluble, released S-pilin (previously correlated with assembly defects) and rare extracellular pili in the mutants?

The precise signal that triggers S-pilin processing is not known, but it has previously been shown to occur concomitantly with reduced levels of piliation (associated with particular pilin subunit antigenic variants (Haas et al., 1987; Swanson et al., 1987)) or absolute defects in pilus expression (Koomey et al., 1987; 1991; Tønjum et al., 1995; Freitag et al., 1995). On the basis of these observations, we proposed that S-pilin processing represents a default pathway of pilin degradation that arises as a consequence of the absence or decreased rate of assembly. However, the signal that leads to S-pilin processing may not be aberrant subunit polymerization but rather a more generalized defect in pilus secretion. In this way, PilQ mutants might still be polymerizing pilin into filaments which are then degraded into S-pilin because they are not extruded from the cell. Unfortunately, this cannot be directly tested because there are no reagents or techniques which enable us to distinguish pilin monomers from polymerized subunits.

If PilQ is, in fact, an essential component of the channel or pore through which pili are extruded, a number of possibilities might explain the presence of rare, extracellular pilus filaments in the mutants tested here. The simplest explanation might be that the truncated PilQ expressed in the mutants retains some function. However, all mutants (including those failing to express any part of the conserved C-terminal domain) fail to form stable multimers and display the same phenotypes, making this an unfavourable explanation. Given the fact that $P$. aeruginosa expresses at least two pIV/PuID homologues (PilQ and XcpQ (Akrim et al., 1993; Martin et al., 1993), gonococci might also have another homologue which partially suppresses the defect in $\mathrm{PilQ}^{-}$mutants. Still, we favour an alternative explanation based on previous observations made in other Type II secretion systems. In Aeromonas hydrophila, Erwinia carotovora and $P$. aenuginosa, mutations that block the second step of the two-step process lead to a defect in export and accumulation of periplasmiclocated toxins or hydrolases. Even under these conditions, however, readily detectable levels of the toxins or hydrolases (estimated to be from $1-6 \%$ of the total amounts) can be detected in culture supernatants (Howard et al., 1993; Reeves et al., 1993; Strom et al., 1991). A similar level of 'leakiness' in the gonococcal mutants could well account for the amounts of pili detected in the PilQ ${ }^{-}$mutants.

The derived amino acid sequence of PilQ from strain MS11 was markedly different from that reported for strain 2686 in the region spanning mature residues 119-186. These alterations, which arise, in part, from use of an altered reading frame, have surprisingly negligible effects on the predicted structural qualities of PilQ except for reducing the number of cysteine moieties from 10 to 7 . The corresponding region of strain $2686 \mathrm{PilQ}$ was noted for its overall basic quality, hydrophilicity and potential for exposure on the surface of the molecule (Tsai et al., 1989), and these qualities are retained in the MS11 molecule. The amino acid-repeat segments of PilQ (encoded by either the MS11 or 2686 alleles) share little similarity to $P$. aeruginosa $\mathrm{PilQ}$ or the other homologues, nor are analogous repeat elements readily apparent in corresponding regions of the homologues. The association of the DNA repeat elements with the sequence discrepancies does raise the possibility that this region may be a hotspot for rearrangements.

Although gonococcal PilQ has generally been portrayed as a highly conserved molecule, tryptic peptide maps

(C) 1995 Blackwell Science Ltd, Molecular Microbiology, 18, 975-986 
have, in fact, documented limited inter-strain heterogeneity, and it was proposed that the 'variation may have resulted from the gain or loss of a peptide component' in the variants versus the reference strain (Hansen and Wilde, 1984). We envisage two ways in which variation within this segment of PilQ might preferentially benefit the organism. The first may be that it represents a form of antigenic variation, as this part of the molecule has been proposed to be exposed at the cell surface. The second relates to the fact that, unlike the case in $P$. aeruginosa pilus expression and the Type II secretion systems, gonococcal PilQ must function in the biogenesis of many different subunits arising by pilin-gene recombination. As such, structural changes in PilQ might finetune the system to accommodate the large repertoire of pilins expressed in vivo.

Strains expressing truncated PilQ represent the first genetically characterized gonococcal competence mutants that retain expression of pilus filaments. Haemophilus influenzae is also naturally competent and DNA uptake is dependent on expression of OrfE (Tomb et al., 1991), which is a homologue of $\mathrm{PilQ}$, suggesting that these homologues may serve similar functions in DNA uptake. Development of competence in $H$. influenzae is associated with the appearance of surface-membrane vesicles termed transformasomes (Kahn et al., 1983), but type IV pili have not been observed in this species.

The findings here strengthen the previously documented conservation found for pilus-biogenesis components in $N$. gonorrhoeae and $P$. aeruginosa. (Lauer et al., 1993). However it is curious that while the other pilus-related proteins share $45 \%$ or greater identity, the two PilQ proteins are only $30 \%$ identical. In spite of this, $P$. aeruginosa is capable of assembling and extruding gonococcal pilin. Even more puzzling is the observation that while the other assembly components have virtually identical predicted isoelectric points, $P$. aeruginosa $\mathrm{PilQ}$ has a predicted $\mathrm{pl}$ of 6.4 , but that predicted for gonococcal PilQ is 9.8. However, the $\mathrm{pl}$ of gonococcal PilQ determined by isolectric focusing was determined to be 7.6 (Hansen and Wilde, 1984). The discrepancies between predicted and observed $\mathrm{pl}$ values for PilQ may reflect some form of post-translational modification or the presence of tightly bound ligands that co-purify with the molecule.

In summary, the product of the pilQ gene, PilQ, plays an essential role in gonococcal pilus expression, and our findings suggest that PilQ functions in the terminal steps of pilus expression rather than in early steps of pilin polymerization, perhaps by acting as a channel for organelle extrusion. Previous studies have concluded that PilQ is exposed at the cell surface (Newhall et al., 1980) and comprises $10 \%$ of the total outer membrane protein mass, but those experiments analysed non-piliated strains and it will be important to repeat those studies using piliated organisms. These and other studies need to be carried out in order to understand precisely how PilQ functions in pilus biogenesis and the expression of related properties, how its structure relates to these functions, and how generally applicable these findings may be to the other seemingly related outer membrane trafficking processes.

\section{Experimental procedures}

\section{Bacterial strains, plasmids, and growth conditions}

Bacterial strains, cloning vectors and cosmid clones containing the pilQ gene are shown in Table 2. Gonococcal strains were propagated on clear solid GC media at $36^{\circ} \mathrm{C}$ in $5 \%$ $\mathrm{CO}_{2}$ or in media lacking agar that had been pre-incubated overnight in $5 \% \mathrm{CO}_{2}$ (Koomey and Falkow, 1987). For examination of secreted proteins, chemically defined media containing free amino acids were used as described previously (Catlin, 1973). E. coli HB101 (Betheseda Research Laboratories) was used in plasmid cloning experiments, and recombinant strains were grown at $37^{\circ} \mathrm{C}$ in Luria-Bertani (LB) medium supplemented with the appropriate antibiotics. The gonococcal (strain VD300) genomic cosmid library used here has been described previously (Koomey and Falkow, 1987).

Table 2. Bacterial strains and plasmids used in this study.

\begin{tabular}{|c|c|c|}
\hline Strain/Plasmid & Relevant characteristics & Source \\
\hline \multicolumn{3}{|c|}{ N. gonormoeae strain } \\
\hline $\mathrm{N} 400$ & $\begin{array}{l}\text { Derived from VD } 300^{\mathrm{a}} \text {. Contains } \\
\text { the recA6 allele (IPTG } \\
\text { inducible) }\end{array}$ & $\begin{array}{l}\text { Freitag et al. } \\
\quad(1995)\end{array}$ \\
\hline $\begin{array}{l}\text { GQ1 } \\
\text { GQ2 }\end{array}$ & $\begin{array}{l}\text { pilQ::m-Tnerm at position } 2516^{\mathrm{b}} \\
\text { pilQ::ermC (using Clal site at } \\
\text { position } 2578^{\mathrm{b}} \text { ) }\end{array}$ & $\begin{array}{l}\text { This work } \\
\text { This work }\end{array}$ \\
\hline GQ3 & $\begin{array}{l}\text { pilQ frameshift mutation at } \\
2578^{\mathrm{b}, \mathrm{c}}\end{array}$ & This work \\
\hline GQ4 & pilQ::m-Tnerm at position $2661^{\text {b }}$ & This work \\
\hline GQ5 & pilQ::m-Tnerm al position $3001^{b}$ & This work \\
\hline GQ6 & pilQ::m-Tnerm at position $3455^{b}$ & This work \\
\hline \multicolumn{3}{|l|}{ Plasmid } \\
\hline pAE8 & $\begin{array}{l}\text { Cosmid clone. Contains the } \\
\text { complete pilQ gene }\end{array}$ & This work \\
\hline pCFI & $\begin{array}{l}\text { Cosmid clone. Contains the } \\
\text { complete pilQ gene }\end{array}$ & This work \\
\hline pAD8 & $\begin{array}{l}\text { Cosmid clone. Contains the } \\
\text { complete pilQ gene }\end{array}$ & This work \\
\hline pBE7 & $\begin{array}{l}\text { Cosmid clone. Contains the } 3^{\prime} \\
\text { end of pilQ gene }\end{array}$ & This work \\
\hline pBluescript SK+ & Cloning vector & Stratagene \\
\hline pQ4 & $\begin{array}{l}\text { 1.3 kb EcoRl pilQ fragment in } \\
\text { pBSK }\end{array}$ & This work \\
\hline pQ9 & $\begin{array}{l}1.6 \mathrm{~kb} \text { Pstl pilQ fragment in } \\
\text { pBSK }\end{array}$ & This work \\
\hline pGo series & $\begin{array}{l}\text { Derived from } 1.6 \mathrm{~kb} P s t \mathrm{I} \\
\text { fragment in pHSS6 }\end{array}$ & This work \\
\hline
\end{tabular}

a. VD300 is a MS11 derivative (Koomey et al., 1987).

b. Derived by transformation using PGQ plasmid DNAs (Fig. 6). Nucleotide sequence positions correspond to those in EMBL/GenBank/DDBJ Nucleotide Sequence Data Libraries under accession number U40596.

c. Contains downstream m-Tnerm insertion at position 3455 . 


\section{General protocols}

Gonococcal mutants were constructed primarily by shuttle mutagenesis using the minitransposon $\mathrm{m}$-Tnerm (a derivative of Tn3) as described by Seifert et al. (1986). The precise sites of insertion were determined by DNA sequencing with primer Tn3L (5'-CTCATGACCAAAATCCC-3') that is complementary to sequences at one end of the transposon. These and other mutations were introduced into $\mathrm{N} 400$ by transformation using linearized plasmid DNAs and selection for erythromycin resistance as detailed in Tønjum et al. (1995). The N400 strain harbours the recA6 allele in which the recA gene is under the control of an inducible tac lac promoter (Freitag et al., 1995). For transformation, RecA expression and recombination functions were transiently induced in this background by IPTG addition to a final concentration of $200 \mu \mathrm{g} \mathrm{ml}^{-1}$ at the same time at which transforming DNA was added.

PCR using N400 genomic DNA and the primers PilQ1 (5'ACGTACCTTGGGATCAGGCTTTGG-3') and PilQ2 (5'-CGGTTGTCCCCCTGAAACCAGTCG-3') was employed to generate the $1.3 \mathrm{~kb}$ pilQ-specific DNA probe which was used to screen the cosmid library by means of colony hybridization. DNA sequencing of the pilQ locus was performed by the dideoxy chain-termination method using a modified form of T7 DNA polymerase (Sequenase 2.0, United States Biochemicals). A $1.3 \mathrm{~kb}$ ECoRI, and a $1.6 \mathrm{~kb}$ Pst fragment were derived from cosmid clone pAE8 and subcloned into pBluescript SK+ (Stratagene), creating $\mathrm{pQ4}$ and pQ9. The complete sequence of both strands of $\mathrm{PQ4}$ and $\mathrm{pQ9}$ was determined using standard vector-based and customized ofigonucleotide primers. To confirm the nucleotide sequence for the segment of the $\mathrm{N} 400$ pilQ gene differing from that of strain 2686, a 700 bp (position 310-1095) DNA fragment was generated by PCR using genomic DNA from N400 and the primers S2 (5'-GCCGATCCTCTGTTGAGC-3') and S3 (5'-CGCTGGCAACAATGTTCA-3'). The nucleotide sequence of this fragment, as well as the complete pilE (pilin subunitencoding) gene of each mutant, was determined by thermocycle sequencing using CircumVent polymerase (New England Biolabs) and customized oligonucleotide primers. DNA and protein sequence data were compiled and analysed by computer using both the MACVECTOR 3.5 (International Biotechnologies Inc.) and the University of Wisconsin Genetics Computer Group (UWGCG) software packages (Devereux et al., 1984).

\section{Gonococcal competence assay}

Competence for natural transformation of gonococcal strains was assessed, as detailed, using chromosomal DNA from spontaneous rifampin- and naladixic acid-resistant mutants of VD300 (Zhang et al., 1992). RecA expression and recombination functions were transiently induced by the addition of IPTG to a final concentration of $200 \mu \mathrm{gml}^{-1}$ at the same time at which transforming DNA was added (see above).

\section{TEM}

Electron microscopy samples were prepared by placing carbon-coated Formvar $3.05 \mathrm{~mm}$ grids (Tousimas Research Corp.) onto individual bacterial colonies which had been propagated for $16 \mathrm{~h}$. Grids were air-dried for $10 \mathrm{~min}$ then floated on a drop of $0.25 \%$ sodium phosphotungstic acid (NaPT) pH7.0 for $30 \mathrm{~s}$ and again air-dried. Grids were examined using a Philips CM-10 Transmission Electron Microscope.

\section{SDS-PAGE and immunoblotting}

Detection of pilin and PilQ in whole-cell lysates and concentrated culture supernatants was performed using rabbit polyclonal pilin-specific antibodies raised against N400 pili (lot 266 , used at a $1: 1000$ dilution) and rabbit polyclonal $P i l Q-$ specific antibodies (provided by $\mathrm{C}$. Wilde III, used at a 1:1000 dilution). Antigen detection was carried out using alkaline-phosphatase-coupled goat anti-rabbit immunoglobulin antibodies (Tago Inc.) and a colorimetric alkaline phosphate substrate. Conditions for sample preparation, SDSPAGE, electroblotting and antigen detection have been described previously (Blake et al., 1984). Pre-stained molecular mass protein standards were employed, obtained from Diversified Biotech and Gibco-BRL.

\section{Acknowledgements}

We are grateful to N. Freitag for help with the EM studies, C. Wilde III for the gonococcal PilQ-specific polyclonal antibodies and $\mathrm{T}$. Tønjum for the meningococcal PilQ-specific antibodies. We also acknowledge the contributions of $P$. Lauer and $\mathrm{R}$. Fox in the original cloning of pilQ and thank $M$. Sandkvist for helpful comments on the manuscript. This work was supported by Public Health Service Grants Al27837 (M.K.); NIH CRC grants M01 RR 00042 (M.K.) and F32 Al09306 (S.D.); and NIH Training Grant T32 8I 07360 (S.D.). M.K. is a Pew Scholar in the Biomedical Sciences.

\section{References}

Akrim, M., Bally, M., Ball, G., Tommassen, J., Teerink, H., Filloux, A., and Lazdunski, A. (1993) Xcp-mediated protein secretion in Pseudomonas aeruginosa: identification of two additional genes and evidence for regulation of $x c p$ gene expression. Mol Microbiol 10: 431-443.

Albano, M., Breitling, R., and Dubnau, D.A. (1989) Nucleotide sequence and genetic organization of the Bacillus subtilis comG operon. J Bacteriol 171: 5386-5404.

Allaoui, A., Sansonetti, P.J., and Parsot, C. (1993) MxiD, and outer membrane protein necessary for the secretion of the Shigella flexneri lpa invasins. Mol Microbiol 7: 59-68.

Blake, M.S., Johnston, K.H., Russell, J.G., and Gotschlich, E.C. (1984) A rapid, sensitive method for detection of alkaline phosphatase-conjugated anti-antibody on Western blots. Anal Biochem 136: 175-179.

Brissette, J.L., and Russel, M. (1990) Secretion and membrane integration of a filamentous phage-encoded morphogenetic protein. J Mol Biol 211: 565-580.

Brissette, J.L., Russel, M., Weiner, L., and Model, P. (1990) Phage shock protein, a stress protein of $E$. coli. Proc Natl Acad Sci USA 87: 862-866.

Carlson, J.H., and Silhavy, T.J. (1993) Signal sequence processing is required for the assembly of LamB trimers in 
the outer membrane of Escherichia coli. J Bacteriol 175: 3327-3334.

Catlin, B.W. (1973) Nutritional profiles of Neisseria gonorrhoeae, Neisseria meningitidis, and Neisseria lactamica in chemically defined media and the use of growth requirements for gonococcal typing. J inf Dis 128: 178-194.

Devereux, J., Haeberli, P., and Smithies, O. (1984) A comprehensive set of sequence analysis programs for the VAX. Nucl Acids Res 12: 387-395.

d'Enfert, C., Reyss, I., Wandersman, C., and Pugsley, A.P. (1989) Protein secretion by Gram-negative bacteria. J Biol Chem 264: 17462-17468.

Freitag, N., Seifert, H.S., and Koomey, M. (1995) Characterization of the pilF-pilD pilus assembly locus of Neisseria gonormoeae. Mol Microbiol 16: 575-586.

Gibbs, C.P., Reimann, B.Y., Schultz, E., Kaufman, A., Haas, R., and Meyer, T.F. (1989) Reassortment of pilin genes in Neisseria gonormoeae occurs by two distinct mechanisms. Nature 338: 651-652.

Giron, J.A., Ho, A.S.Y., and Schoolnik, G.K. (1991) An inducible bundle-forming pilus of enteropathogenic Escherichia coli. Science 254: 710-713.

Haas, R., and Meyer, T.F. (1986) The repertoire of silent pilus genes in Neisseria gonormoeae: evidence for gene conversion. Cell 44: 107-115.

Haas, R., Schwarz, H., and Meyer, T.F. (1987) Release of soluble pilin antigen coupled with gene conversion in Neisseria gonorrhoeae. Proc Natl Acad Sci USA 84: 90799083.

Hansen, M.V., and Wilde, C.E. (1984) Conservation of peptide structure of outer membrane protein-macromolecular complex from Neisseria gonormoeae. Infect Immun 43: 839845.

Heckels, J.E. (1989) Structure and function of pili of pathogenic Neisseria species. Clin Microbiol Rev 2: S66-S73.

Hermodson, M.A., Chen, K.C., and Buchanan, T.M. (1978) Neisseria pili proteins: amino-terminal amino acid sequences and identification of an unusual amino acid. Blochemistry 17: $442-445$.

Howard, S.P., Critch, J., and Bedi, A. (1993) Isolation and analysis of eight exe genes and their involvement in extracellular protein secretion and outer membrane assembly in Aeromonas hydrophila. J Bacteriol 175: 6695-6703.

Huang, H.C., He, S.Y., Bauer, D.W., and Collmer, A. (1992) The Pseudomonas syringae pv. syringae 61 hrpH product, an envelope protein required for elicitation of the hypersensitive response in plants. J Bacteriol 174: 6878-6885.

Jonsson, A.-B., Nyberg, G., and Normark, S. (1991) Phase variation of gonococcal pili by frameshift mutation in pilC, a novel gene for pilus assembly. EMBO $J$ 10: 477-488.

Kahn, M., Barany, F., and Smith, H.O. (1983) Transtormasomes: specialized membranous structures that protect DNA during Haemophilus transformation. Proc Natl Acad Sci USA 80: 6927-6931.

Kazmierczak, B.I., Mielke, D.L., and Russel, M. (1994) pIV, a filamentous phage protein that mediates phage export across the bacterial cell envelope, forms a multimer. $J \mathrm{Mol}$ Biol 238: 187-198.

Koomey, J.M., and Falkow, S. (1987) Cloning of the recA gene of Neisseria gonorrhoeae and construction of gonococcal RecA ${ }^{-}$mutants. J Bacteriol 169: 790-795.
Koomey, M., Gotschlich, E.C., Robbins, K., Bergstrom, S., and Swanson, J. (1987) Effects of recA mutations on pilus antigenic variation and phase transitions in Neisseria gonorrhoeae. Genetics 117: 391-398.

Koomey, M., Bergstrom, S., Blake, M., and Swanson, J. (1991) Pilin expression and processing in pilus mutants of Neisseria gonorrhoeae: critical role of Gly -1 in assembly. Mol Microbiol 5: 279-287.

Lauer, P., Albertson, N.H., and Koomey, M. (1993) Conservation of genes encoding components of a type IV pilus assembly/two-step protein export pathway in Neisseria gonorrhoeae. Mol Microbiol 8: 357-368.

Lory, S. (1992) Determinants of extracellular protein secretion in Gram-negative bacteria. J Bacteriol 174: 3423-3428.

Martin, P.R., Hobbs, M., Free, P.D., Jeske, Y., and Mattick, J.S. (1993) Characterization of pilQ, a new gene required for the biogenesis of type 4 fimbriae in Pseudomonas aeruginosa. Mol Microbiol 9: 857-868.

Michiels, T., and Cornelis, G.R. (1991) Secretion of hybrid proteins by the Yersiniae Yop export system. J Bacteriol 173: $1677-1685$.

Newhall, W.J., Wilde, C.E., Sawyer, W.D., and Haak, R.A. (1980) High-molecular-weight antigenic protein complex in the outer membrane of Neisseria gonormoeae. Infect Immun 27: 475-482.

Nunn, D., Bergman, S., and Lory, S. (1990) Products of three accessory genes, pilB, pilC and pilD, are required for biogenesis of Pseudomonas aeruginosa pili. J Bacteriol 172: 2911-2919.

Possot, O., d'Enfert, C., Reyss, I., and Pugsley, A.P. (1992) Pullulanase secretion in Escherichia coli $\mathrm{K}-12$ requires a cytoplasmic protein and a putative polytopic cytoplasmic membrane protein. Mol Microbiol 6: 95-105.

Pugsley, A.P. (1992) Superfamilies of bacterial transport systems with nucleotide binding components. Symp Soc Gen Microbiol 47: 223-248.

Pugsley, A.P. (1993) The complete general secretory pathway in Gram-negative bacteria. Microbiol Rev 57: 50-108.

Reeves, P.J., Whitcombe, D., Wharman, S., Gibson, M., Allison, G., Bunce, N., Barallon, R., Douglas, V., Mulholland, V., Stevens, S., Walker, D., and Salmond, G.P.C. (1993) Molecular cloning and characterization of 13 out genes from Erwinia carotovora subspecies carotovora: genes encoding members of the general secretion pathway (GSP) widespread in Gram-negative bacteria. Mol Microbiol 8: $443-456$.

Rudel, T., van Putten, J.P.M., Gibbs, C.P., Hass, R., and Meyer, T.F. (1992) Interaction of two variable proteins (PilE and PilC) required for pilus-mediated adherence of Neisseria gonorrhoeae to human epithelial cells. Mol Microbiol 6: 3439-3450.

Russel, M. (1994) Mutants at conserved positions in gene IV, a gene required for assembly and secretion of filamentous phages. Mol Microbiol 14: 357-369.

Russel, M., and Kasmierczak, B. (1993) Analysis of the structure and subcellular location of filamentous phage plV. $J$ Bacteriol 175: 3998-4007.

Salmond, G.P.C., and Reeves, P.J. (1993) Membrane traffic wardens and protein secretion in Gram-negative bacteria. Trends Biochem Sci 18: 7-12.

Seifert, H.S., Chen, E.Y., So, M., and Heffron, F. (1986) 
Shuttle mutagenesis: a method of transposon mutagenesis for Saccharomyces cerevisiae. Proc Natl Acad Sci USA 83: 735-739.

Seifert, H.S., Ajioka, R.S., Paruchuri, D., Heffron, F., and So, M. (1990) Shuttle mutagenesis of Neisseria gonorrhoeae: pilin null mutations lower DNA transformation competence. $J$ Bacteriol 172: 40-46.

Shaw, C.E., and Taylor, R.K. (1990) Vibrio cholerae 0395 tcpA pilin gene sequence and comparison of the predicted protein structural features to those of type IV pilins. Infect Immun 58: 3042-3049.

Strom, M.S., Nunn, D., and Lory, S. (1991) Multiple roles of the pilus biogenesis protein pilD: involvement of pilD in excretion of enzymes from Pseudomonas aeruginosa. $J$ Bacteriol 173: 1175-80.

Swanson, J. (1973) Studies on gonococcus infection IV. Pili: their role in attachment of gonococci to tissue culture cells. $J$ Exp Med 137: 571-589.

Swanson, J. (1978) Studies on gonococcus infection XII. Colony color and opacity variants of gonococci. Infect Immun 19: 320-331.

Swanson, J., and Koomey, J.M. (1989) Mechanisms for variation of pili and outer membrane protein II in Neisseria gonorrhoeae. In Mobile DNA. Berg, D., and Howe, M. (eds). Washington, DC: ASM, pp. 743-761.

Swanson, J., Kraus, S.J., and Gotschlich, E.C. (1971) Studies on gonococcus infection I. Pili and zones of adhesion: their relation to gonococcal growth patterns. J Exp Med 134: 886-906.
Swanson, J., Robbins, K., Barrera, O., Corwin, D., Boslego, J., Ciak, J., Blake, M., and Koomey, J.M. (1987) Gonococcal pilin variants in experimental gonnorhoea. $J$ Exp Med 165: 1344-1357.

Tomb, J., El-Hajj, H., and Smith, H.O. (1991) Nucleotide sequence of a cluster of genes involved in the transformation of Haemophilus influenzae Rd. Gene 104: 1-10.

Tønjum, T., Freitag, N.E., Namork, E., and Koomey, M. (1995) Identification and characterization of pilG, a highly conserved pilus assembly gene in pathogenic Neisseria. Mol Microbiol 16: 451-464.

Tsai, W., Larsen, S.H., and Wilde, C.E.I. (1989) Cloning and DNA sequence of the omc gene encoding the outer membrane protein-macromolecular complex from Neisseria gonorrhoeae. Infect Immun 57: 2653-2659.

Virji, M., Heckels, J.E., and Watt, P.J. (1983) Monoclonal antibodies to gonococcal pili: studies on antigenic determinants on pili from variants of strain P9. J Gen Microbiol 129: 1965-1973.

Whitchurch, C.B., Hobbs, M., Livingston, S.P., Krishnapillai, V., and Mattick, J.S. (1991) Characterisation of a Pseudomonas aeruginosa twitching motility gene and evidence for a specialized protein export system widespread in eubacteria. Gene 101: 33-44.

Zhang, Q.Y., DeRyckere, D., Lauer, P., and Koomey, M. (1992) Gene conversion in Neisseria gonorhoeae: evidence for its role in pilus antigenic variation. Proc Natl Acad Sci USA 89: 5366-5370. 